\title{
Anticonceptivos orales en pacientes con psicopatología alimentaria: implicaciones etiopatogénicas y terapéuticas.
}

Oral contraceptives in patients with eating psychopathology: Etiopathogenic and therapeutic implications.

\author{
Álvaro Frías Ibáñez a , María Vázquez Costa b. \\ ${ }^{a}$ Centro de salud mental infantojuvenil. Hospital de Mataró, Mataró, Barcelona, España. ${ }^{b}$ Centro de salud \\ mental infantojuvenil. Hospital de Mataró, Mataró, Barcelona, España.
}

Correspondencia: Álvaro Frías Ibáñez(alvarofriasibanez@gmail.com)

Recibido: 07/10/2011; aceptado: 19/02/2012

RESUMEN: Introducción. El papel de los anticonceptivos orales (ACO) en el ámbito de la psiquiatría apenas ha sido objeto de atención clínica. En concreto, existe una ínfima literatura científica respecto a su rol en la etiopatogenia y terapéutica de la psicopatología alimentaria.

Caso Clínico. Se expone el caso de una mujer de 16 años que desarrolla una anorexia nerviosa tras la administración de un ACO para el tratamiento de un hirsutismo hiperandrogénico leve. Con la implementación de terapia cognitivo-conductual y psicofarmacológica (ISRS) se objetivó una restauración del peso, encapsulándose las cogniciones anorexígenas y remitiendo las conductas alimentarias disfuncionales. Conclusiones. El estudio de los ACO en el ámbito de la psicopatología alimentaria se halla en fase embrionaria, postulándose la regulación androgénica como principal variable mediadora de esta asociación. En pacientes con anorexia nerviosa, es preceptivo implementar investigaciones longitudinales, dadas las pruebas preliminares que sugieren la participación de los ACO como factor de riesgo psicopatológico, ya sea a través de la disminución de los niveles de testosterona y/o del mayor temor a engordar entre aquellas usuarias con ganancia ponderal tras su administración.

PALABRAS CLAVE: anticonceptivos orales, anorexia nerviosa, testosterona.
ABSTRACT: Introduction. The role of oral contraceptives (OC) in psychiatry illness has been object of little attention. In particular, there is scarce scientific literature regarding its influence in the pathogenesis and treatment of eating psychopathology.

Clinical Case. We describe the case of a 16 yearsold woman, who developed an anorexia nervosa after the administration of an OC for the treatment of a mild hyperandrogenic hirsutism. After the implementation of cognitive-behavioral and psychopharmacological (SSRI) interventions, weight restoration was attained, disappearing anorectic cognitions and dysfunctional eating behaviors.

Conclusions. The study of OC in eating psychopathology is in an early stage, being androgenic regulation postulated as main factor mediating this association. In patients with anorexia nervosa, is mandatory carrying out longitudinal research, given preliminary evidence suggesting the involvement of $\mathrm{OC}$ as a risk factor, either through decreased levels of testosterone or greater fear of gaining weight among those users with weight gain after its administration.

KEY WORDS: oral contraceptives, anorexia nervosa, testosterone. 


\section{Introducción}

El papel de los anticonceptivos orales (ACO) en el ámbito de la psiquiatría apenas ha sido objeto de atención clínica (1). En concreto, existe una ínfima literatura respecto a su rol en la etiopatogenia y/o terapéutica de la psicopatología alimentaria (2).

\section{Caso Clínico}

Motivo de consulta

Se expone el caso de una mujer de 16 años derivada al centro de salud mental infanto-juvenil del área para valoración por "probable trastorno conducta alimentaria (TCA)".

Historia del problema

La paciente refiere comportamiento alimentario disfuncional de seis meses de evolución tras ganancia ponderal (5 kilos) secundaria a tratamiento con ACO. Presenta restricción de la ingesta, atracones periódicos (tres/semana), así como exacerbación de la actividad física (una hora footing/día). Señala intenso temor a engordar y distorsión de la imagen corporal (vientre), motivando ello comprobaciones compulsivas en espejos y aislamiento social. Se objetiva pérdida ponderal de $13 \mathrm{~kg}$ en el último semestre (índice masa corporal (IMC) 16,94).

\section{Psicobiografía}

Fue fruto de un parto sin incidencias. Desarrollo normativo de los hitos madurativos. Descrita desde la infancia como una persona "independiente y reservada". Cuando tenía 11 años, su madre falleció por cáncer de páncreas, llevando a cabo un proceso de duelo normativo. Buena relación con su padre y hermana. Círculo de amigas estable. Niega consumo de tóxicos. Buen rendimiento académico. A partir de la adolescencia, presenta rumiaciones obsesivoides en relación al crecimiento del vello (piernas), siendo este incremento apenas perceptible para los familiares. Realiza sesiones de depilación semanales, comprobaciones compulsivas de sus extremidades y evitación de la exposición corporal (nunca muestra sus piernas). Es diagnosticada a los 16 años de hirsutismo hiperandrogénico leve, iniciando tratamiento con ACO $(2,0 \mathrm{mg}$ de acetato de ciproterona y $0,035 \mathrm{mg}$ de etinilestradiol). Remite con ello el tenue aumento de vello, describiendo adicionalmente un aumento del apetito durante los primeros meses de administración. 
NOTAS CLÍNICAS

Exploración clínica

Durante la entrevista se hallaba consciente y orientada. Discurso reactivo, destacando intenso miedo a engordar, así como distorsiones de la imagen corporal (vientre), con continuos rituales de reaseguramiento ante el espejo. Asimismo, refiere comprobaciones corporales recurrentes ante el temor al crecimiento del vello, pese a su remisión. Ánimo hipotímico. Hiporexia, con atracones periódicos. Conciencia parcial de enfermedad.

Pruebas complementarias

- Analítica previa a ACO: testosterona=0.71 (0.28-11), dehidroepiandrosterona $($ DHEA $)=10.1(1-8)$

- Analítica tras ACO: testosterona=0.65, DHEA=7.14

Diagnóstico

Anorexia nerviosa, tipo purgativo F50.0

Trastorno dismórfico corporal F45.2

Tratamiento y evolución

Se inició tratamiento ambulatorio intensivo (semanal), implementándose psicoterapia conductual a través de "control estimular" (reducir actividad física) y "exposición con prevención de respuesta" (aumentar las cantidades ingeridas sin atracones). Paralelamente, se ejecutó un programa de "activación conductual" (salir con amigos, pasear) y un módulo "psicoeducativo" familiar. En cuanto al tratamiento psicofarmacológico, se administró Fluoxetina 20 1-0-0.

Tras siete meses de seguimiento, la paciente presentaba conductas disfuncionales residuales (comprobaciones ocasionales en espejo), objetivándose un peso saludable (IMC 21,14) y manifestando un encapsulamiento de las cogniciones anorexígenas. Remitido el TCA y estabilizada anímicamente, actualmente se está abordando su sintomatología dismorfofóbica con terapia cognitivo-conductual.

\section{Conclusiones}

El mecanismo de acción de los ACO implica la inhibición de la síntesis de esteroides gonadales femeninos, liberando para ello dosis menores de análogos sintéticos (p.e. etinilestradiol). Adicionalmente, muchos ACO disminuyen los niveles de esteroides gonadales masculinos, con frecuencia administrando hormonas sintéticas antiandrógenas (p.e. acetato de ciproterona) (2). A nivel interventivo, los 
ACO han demostrado ser una terapéutica eficaz para la remisión y prevención de diversas patologías médicas, incluido el hirsutismo hiperandrogénico leve (3).

En el ámbito de la psiquiatría, los ACO apenas han sido objeto de interés clínico, estando ligados principalmente al tratamiento del trastorno disfórico premenstrual, cuadro nosológico donde se han obtenido resultados concluyentes sobre su efectividad (1). Por otro lado, no existen pruebas consistentes sobre su relevancia como factor de riesgo psicopatológico, a diferencia de los anticonceptivos depot de progesterona, evidenciándose con estos últimos una mayor incidencia de sintomatología depresiva (4).

En este contexto general, se ha ido desarrollando una incipiente investigación empírica encaminada a determinar el papel etiopatogénico y/o terapéutico de los ACO en los TCA, hecho sustentado sobre la base de los correlatos gonadales hallados en estos pacientes psiquiátricos. En relación a ello, se ha constatado que, respecto a la población sana, las personas con anorexia nerviosa presentan niveles inferiores de estrógenos y testosterona, no existiendo hallazgos concluyentes sobre una menor síntesis de DHEA (5-6). A nivel neuroendocrino, se ha asociado este déficit estrogénico con diversas complicaciones médicas (p.e.osteopenia), mientras que la disminución de testosterona, con las cogniciones anorexígenas (7). Por lo que respecta a la bulimia nerviosa, algunas investigaciones han obtenido niveles de testosterona superiores a los sujetos sin patología, relacionando este exceso androgénico con el comportamiento bulímico (8).

En el ámbito interventivo, estos hallazgos neuroendocrinos en muestras TCA han estimulado el uso terapéutico de los ACO con dichos pacientes. Por una parte, se ha promovido su utilización en el tratamiento de la osteopenia secundaria en sujetos anoréxicas, arrojando resultados inconsistentes sobre su efectividad (9), a diferencia de las evidencias positivas en mujeres perimenopáusicas (10). Por otro lado, se ha postulado su administración en pacientes bulímicas, existiendo pruebas preliminares que apoyan la remisión psicopatológica con ACO antiandrogénicos (11).

A nivel etiopatogénico, el papel de los ACO como potencial factor de riesgo para la psicopatología alimentaria proviene principalmente de estudios transversales implementados en muestras ginecológicas, sugiriéndose variables neuroendocrinas y psicológicas que pudieran estar mediando esta relación. Respecto a las hipótesis biomédicas, se ha observado que aquellas mujeres con más efectos secundarios físicos y emocionales derivados de los ACO presentan un mayor riesgo de manifestar cogniciones TCA (temor a engordar y distorsiones perceptivas), planteando una mayor "sensibilidad gonadal" en este subgrupo de mujeres con ACO (2). Por otro lado, se ha hallado que las pacientes anoréxicas en tratamiento con ACO antiandrogénicos presentan los niveles más bajos de testosterona de dicha muestra (11), sugiriéndose el tratamiento de este trastorno mental mediante terapia androgénica sustitutiva, dada la asociación entre déficit androgénico y cognicio- 
nes anorexígenas (7). Desde una perspectiva más psicologicista, otras líneas de investigación han evaluado la ligazón entre cogniciones TCA y aumento de apetito/peso secundario a ACO. Sobre esta cuestión, algunos estudios apuntan que las mujeres en tratamiento presentan un incremento adicional del hambre durante la fase menstrual (12-13), no comportando ello una mayor saliencia de cogniciones TCA en este período del ciclo reproductor (12). Del mismo modo, tampoco se ha corroborado una asociación específica entre ganancia ponderal y psicopatología alimentaria entre aquellas pacientes evaluadas en relación a los efectos secundarios de los ACO (2). De hecho, salvo en sujetos particulares (14), no existen evidencias concluyentes que apoyen un incremento significativo en el IMC en mujeres con ACO (4). Considerando estos últimos datos, el caso descrito adquiere mayor relevancia al señalar una relación directa entre aumento de peso secundario a ACO y anorexia nerviosa.

En conclusión, el estudio de los ACO en el ámbito de la psicopatología alimentaria se halla en fase embrionaria, postulándose la regulación androgénica como principal variable mediadora de esta asociación. Por una parte, deben estimularse ensayos clínicos con ACO antiandrogénicos en pacientes bulímicas, considerando los correlatos neuroendocrinos hallados en estos sujetos. Por otro lado, es preceptivo implementar investigaciones longitudinales en pacientes anoréxicas con el fin de determinar la relevancia etiopatogénica de los ACO, dadas las pruebas preliminares que sugieren su implicación a través de la disminución de los niveles de testosterona y/o del mayor temor a engordar en aquellas usuarias con ganancia ponderal. A nivel preventivo, estos últimos hallazgos plantean la importancia de llevar a cabo un seguimiento periódico de aquellas usuarias con psicopatología alimentaria subclínica, especialmente si ya presentaban antecedentes de distorsiones de la imagen corporal.

BIBLIOGRAFÍA:

(1) Freeman EW. Therapeutic management of premenstrual syndrome. Expert Opin Pharmacother 2010;11(17):2879-89.

(2) Bird JL, Oinonen KA. Elevated eating disorder symptoms in women with a history of oral contraceptive side effects. Arch Womens Ment Health 2011;14(4):345-53.

(3) Sahin Y, Bayram F, Kelestimur F, Muderris I. Comparison of cyproterona acetate plus ethinyl estradiol and finasteride in the treatment of hirsutism. J Endocrinol Invest 1998;21(6):348-52.

(4) Grossman, N. Managing adverse effects of hormonal contraceptives. American Family Physician 2010;82(12):1499-506.

(5) Lawson EA, Misra M, Meenaghan E, Rosenblum L, Donoho A, Herzog D., et al. Adrenal glucocorticoid and androgen precursor dissociation in anorexia nervosa. J Clin Endocrinol Metab 2009;94(4):1367-71. 
(6) Estour B, Germain N, Diconne E, Frere D, Cottet-Emard JM, Carrot G, et al. Hormonal profile heterogeneity and short-term physical risk in restrictive anorexia nervosa. J Clin Endocrinol Metab 2010;95(5):2203-10.

(7) Miller KK, Wexler TL, Zha AM, Lawson EA, Meenaghan EM, Misra M., et al. Androgen deficiency: association with increased anxiety and depression symptoms severity in anorexia nervosa. J Clin Psychiatry 2007;68(6):959-65.

(8) Naessen S, Carlstrom K, Bystrom B Pierre Y, Hirschberg AL. Effects of an antiandrogenic oral contraceptive on appetite and eating behavior in bulimic women. Psychoneuroendocrinology 2007;32(5): 548-54.

(9) Strokosch GR, Friedman AJ, Wu SC, Kamin M. Effects of an oral contraceptive (norgestimate/ethinyl estradiol) on bone mineral density in adolescent females with anorexia nervosa: a doubleblind, placebo-controlled study. J Adolesc Health 2006;39:819-27.

(10) Liu SL, Lebrun CM. Effect of oral contraceptives and hormone replacement therapy on bone mineral density in premenopausal and perimenopausal women: a systematic review. Br J Sports Med 2006;40:11-24.

(11) Miller KK, Lawson EA, Mathur V, Wexler TL, Meenaghan E, Misra M., et al. Androgens in women with anorexia nervosa and normal-weight women with hypothalamic amenorrhea. J Clin Endocrinol Metab 2007;92:1334-9

(12) McVay MA, Copeland AL, Geiselman PJ. Eating disorder pathology and menstrual cycle fluctuations in eating variables in oral contraceptive users and non-users. Eating Behaviors 2011;12:49-55.

(13) Bancroft J, Rennie D. The impact of oral contraceptives on the experience of perimenstrual mood, clumsiness, food craving and other symptoms. J Psychosom Res 1993;37:195-202.

(14) Meier CR. Health risks of oral contraceptives. Ther Umsch 2011;68(6):345-52. 